\title{
Wer gute Pflege will, muss auch Geld investieren
}

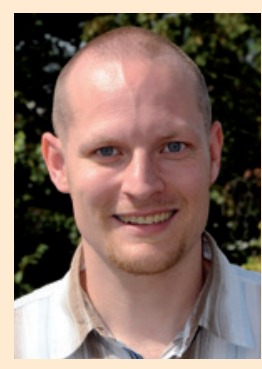

Christian Schäfer, Stuttgart
Es ist schon längst überfällig, aber immerhin begreifen anscheinend immer mehr Politiker in Deutschland die Tragweite und Dringlichkeit des Problems: Überlastete Mitarbeiter in den Kliniken bzw. sich verschlechternde Arbeitsbedingungen, zu geringe Investitionen und ein sich immer stärker aufbauender Fachkräftemangel u.a. in der Pflege haben das Potenzial, eine adäquate Patientenversorgung entscheidend zu gefährden. V.a. das Extrapolieren der Bevölkerungszusammensetzung auf die nächsten Jahrzehnte zeigt, dass man aufgrund eines weiteren Rückgangs der Personenzahl im Erwerbsalter und eines gleichzeitigen Anstiegs der Zahl von alten Menschen deutlich gegensteuern muss.

Derzeit diskutiert die Große Koalition bzgl. des sog. Pflegestellenprogramms eine Verdoppelung der Mittel auf 1,32 Milliarden Euro, wie die gesundheitspolitische Sprecherin der SPD-Bundestagsfraktion Hilde Mattheis gegenüber der Ärzte Zeitung sagte. Das Ziel sei, für die Beschäftigten in den Krankenhäusern v.a. in der Pflege bessere Arbeitsbedingungen zu schaffen und somit Personal inner- oder außerhalb der DRGs (Diagnosis Related Groups: diagnosebezogene Fallgruppen) besser abzubilden. Die Krankenhäuser müssten sich hierbei mit $10 \%$ an den Kosten beteiligen und nachweisen, dass sie seit dem Jahr 2015 keine Stellen abgebaut haben. Wie Bundesgesundheitsminister Hermann Gröhe (CDU) betonte, sollen etwa 6300 neue Pflegestellen in den Krankenhäusern Deutschlands geschaffen werden. Das ist auch dringend notwendig, denn es fehlen laut ver.di dort 70000 Pflegekräfte. Und das ist ja erst der Anfang - zukünftig könnte sich die Zahl weiter erhöhen. Von daher können diese möglichen Investitionen eine hoffentlich nun beginnende Entwicklung in der politischen Landschaft sein, die tatsächlich konkret die Bedingungen für die Pflege verbessern. Denn dies ist eine der wichtigsten Grundlagen dafür, um eine gute Pflege zu ermöglichen - Politiker drehen endlich mehr und mit höherer Wahrscheinlichkeit an den richtigen Stellschrauben.

Speziell in der nephrologischen Pflege zeigen sich nach der Kürzung der Dialyse-Sachkosten-Pauschale und den darauf folgenden Sparmaßnahmen diese Probleme deutlich: Die Betreuung der meist multimorbiden Patienten enthält ein komplexes Geflecht aus Einschätzungen und Maßnahmen, bei der auch die Pflege eine sehr wichtige Rolle spielt. Auch das Verständnis der komplexen Nierenersatzverfahren und Dialysetechniken erfordert eine intensive und längere Beschäftigung mit der Thematik. Der Fachkräftemangel und somit das Fehlen von eingearbeiteten, erfahrenen Mitarbeitern sowie der immer größer werdende Zeitmangel wirken sich hier besonders stark aus. Zudem befürchtet die AfnP (Arbeitsgemeinschaft für nephrologisches Personal e. V.), dass durch die Einstellung von unerfahrenen, nicht speziell fort- und weitergebildeten Pflegekräften die Behandlungsqualität sinkt und somit Komplikationen bei den Patienten zunehmen. Beachten Sie hierzu den Artikel ab Seite 288 in dieser Ausgabe der Dialyse aktuell.

Mit alternden Nierenkranken und somit einem Patientenklientel, das in Zukunft zahlenmäßig und vom Anteil her wahrscheinlich deutlich zunehmen wird, beschäftigen sich die kompetenten Autoren der Beiträge zum Schwerpunkt dieser Ausgabe. Sie können ab Seite 297 die interessanten Artikel zum Thema „Geriatrische Nephrologie“ lesen. Ich wünsche Ihnen eine angenehme Lektüre der Dialyse aktuell 6/2015! 